OPINIÓN

\title{
Restauración ecológica para aumentar la provisión de agua como un servicio ecosistémico en cuencas forestales del centro-sur de Chile
}

\author{
Ecological restoration for water yield increase as an ecosystem \\ service in forested watersheds of south-central Chile \\ Christian Little ${ }^{a, c *}$, Antonio Lara ${ }^{b, c}$

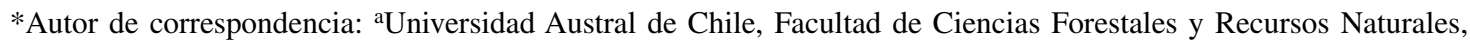 \\ Escuela de Graduados, casilla 567, Valdivia, Chile, tel.: 56-63-293038, little.christian@ gmail.com \\ bUniversidad Austral de Chile, Facultad de Ciencias Forestales y Recursos Naturales, Instituto de Silvicultura, Valdivia, Chile. \\ cFundación Centro de los Bosques Nativos FORECOS, Valdivia, Chile.
}

\begin{abstract}
SUMMARY
The relationship between ecosystem and ecosystem services is revisited in order to integrate these concepts to water yield, as an important service provided by watersheds for human welfare. We propose that in watersheds disturbed by human activities, water yield can by re-established using the ecological restoration principles and practices. We also suggest a reference image integrating social, economic and environmental dimensions, and at the same time increasing research and education capacities. We discuss research progress in Chile regarding these issues referred to a study in small watersheds in the Reserva Costera Valdiviana, projected as a Long Term Ecosystem Research site. We conclude that it is necessary to improve both, scientific knowledge and outreach programs about ecosystem services, through a transdisciplinary approach and methods.
\end{abstract}

Key words: streamflow, resilience, reference image, Reserva Costera Valdiviana, valdivian rainforest.

\section{RESUMEN}

Se revisa la relación entre ecosistemas y servicios ecosistémicos, integrándolos a la provisión de agua en cuencas hidrográficas, uno de los servicios más importantes para el bienestar de las personas. Se propone que en cuencas alteradas por actividades antropogénicas, la provisión de agua puede ser recuperada utilizando los principios y prácticas de la restauración ecológica; y se propone una imagen de referencia que integre aspectos ambientales, económicos y sociales, e incluya el incremento de la investigación científica y educación. Se discuten algunos avances en Chile referidos a un estudio a escala de pequeñas cuencas en la Reserva Costera Valdiviana, que se proyecta como una estación de estudios ecosistémicos de largo plazo. Se concluye que es necesario avanzar urgentemente en el conocimiento científico y su divulgación social, respecto a los servicios ecosistémicos, incorporando enfoques de trabajo y metodologías transdisciplinarias.

Palabras clave: caudales, resiliencia, imagen de referencia, Reserva Costera Valdiviana, bosque valdiviano.

\section{INTRODUCCIÓN}

Durante las últimas décadas, el centro-sur de Chile ha tenido una serie de transformaciones del paisaje expresada en cambios del uso del suelo y con ello en la cobertura de bosques (Echeverría et al. 2006). Estos drásticos cambios e intensa presión por el uso del territorio han resultado en la incompatibilidad entre diferentes bienes y servicios que proveen los ecosistemas (Lara et al. 2003, 2008, 2009), observándose con mayor frecuencia conflictos por el uso de los recursos naturales. Diversos ejemplos ilustran esta situación: la implementación de proyectos hidroeléctricos en áreas con potencial turístico, la deforestación y degradación de bosques naturales en áreas con alta biodiversidad y la producción de madera basada en plantaciones de rápido crecimiento con la provisión de agua.

Estudios recientes demuestran la posibilidad de compatibilizar los objetivos de desarrollo socioeconómicos con la oferta de servicios ecosistémicos (Rey Benayas et al. 2009), planteándose la necesidad de desarrollar políticas públicas que incorporen una producción balanceada entre los diversos bienes y servicios que proveen los ecosistemas. Por ejemplo, la producción maderera basada en plantaciones de rápido crecimiento y la captura de carbono (Farley et al. 2005, Jackson et al. 2005) o la producción maderera con la provisión de agua en cuencas forestales (Lara et al. 2009). En este contexto, los objetivos de la presente opinión son entregar una conceptualización relativa 
a este último servicio ecosistémico, proponer acciones para su recuperación en cuencas perturbadas y, finalmente, divulgar un experimento a escala de pequeñas cuencas en la Reserva Costera Valdiviana ${ }^{1}$ que se proyecta como una estación experimental de largo plazo.

\section{SERVICIO ECOSISTÉMICO DE PROVISIÓN DE AGUA EN CUENCAS FORESTALES DEL CENTRO-SUR DE CHILE}

En el centro-sur de Chile los bosques nativos proveen diversos servicios ecosistémicos (Lara et al. 2003, 2009, Oyarzún et al. 2005). Sin embargo, como consecuencia de prácticas forestales inadecuadas, incendios, sustitución por plantaciones forestales de Pinus radiata D. Don o Eucalyptus spp. y habilitación agropecuaria (Lara et al. 2003, Echeverría et al. 2006), se ha observado un deterioro o disminución en la oferta de algunos de ellos, tales como diversidad biológica (Echeverría et al. 2006) y provisión de agua en calidad y cantidad (Oyarzún et al. 2007, Little et al. 2008, 2009, Lara et al. 2009).

Respecto a la provisión de agua como servicio ecosistémico, es en una cuenca hidrográfica donde se conjugan todos los elementos que conforman un ecosistema y ocurren los procesos que regulan la oferta de este servicio ecosistémico. Por lo tanto, la estructura, funcionamiento y estado de conservación de la cuenca determinarán el bienestar logrado en cada una de las actividades que demanda el servicio, como, por ejemplo, para la producción de agua potable, hidroelectricidad, salmonicultura, pesca recreativa, etc. (Lara et al. 2008, 2009).

En orden de importancia, las variables que impactan la oferta del servicio ecosistémico provisión de agua en cuencas forestales son: a) las precipitaciones que las abastecen (tipo, cantidad, intensidad, estacionalidad y variabilidad) (Ward y Trimble 2003); b) su geomorfología y geología (área de drenaje, pendiente, tipo y profundidad de suelo, etc.) que almacena y conduce el agua de las precipitaciones hacia las napas freáticas, acuíferos, arroyos, ríos, lagos, etc.; y c) su cobertura vegetal así como el uso del suelo y su manejo que cambia las tasas de evapotranspiración (Angelstam et al. 2005, Huber et al. 2008), modificando los caudales de flujo base y punta (Calder 1992, Scott y Prinsloo 2008, Lara et al. 2009). Estudios recientes, desarrollados para cuencas forestales del centro-sur de Chile, demuestran que un aumento en $10 \%$ de plantaciones forestales de rápido crecimiento en pequeñas cuencas (140-1.900 ha) reducen en un 20,4\% los caudales de verano (Lara et al. 2009). En zonas con déficit hídrico cubiertas por plantaciones, dicha cifra pudiera ser aún mayor, considerando las altas tasas de evapotranspiración de estas plantaciones, comparada con otros usos de suelo

\footnotetext{
1 Área protegida privada abierta al público, de propiedad de The
} Nature Conservancy. http://www.reservacosteravaldiviana.cl
(Farley et al. 2005), tal como en la zona central de Chile donde sólo un $5 \%$ de la precipitación queda disponible para la recarga de acuíferos y generación de escorrentía (Huber et al. 2008). Little et al. (2009) documentan que el reemplazo de bosques nativos por plantaciones forestales de rápido crecimiento en zonas con baja precipitación ha provocado una caída de los caudales de verano en un $42,7 \%$ y $31,9 \%$ en dos cuencas de gran tamaño (25.250 y 70.770 ha, respectivamente).

\section{RESTAURACIÓN ECOLÓGICA Y RECUPERACIÓN DEL SERVICIO ECOSISTÉMICO PROVISIÓN DE AGUA}

El cambio en la oferta del servicio ecosistémico provisión de agua, como una consecuencia de diversos procesos de degradación y funcionamiento de las cuencas, aún se presenta con poca claridad y, por lo tanto, ha tenido un escaso impacto en el desarrollo de políticas públicas (Locatelli y Vignola 2009). Hasta ahora se ha visto como un servicio ecosistémico a conservar o mantener en las cuencas, pero no a recuperar, lo cual implicará importantes desafíos científico-tecnológicos. Algunas acciones antropogénicas, como la construcción de represas, diques o canales para el desvío de los cursos de agua, han sido capaces de regular la oferta de agua para los períodos de escasez y mayor demanda. Sin embargo, existen otras actividades con el mismo objetivo que se centran en aumentar la oferta del servicio ecosistémico provisión de agua en las cuencas, las cuales hasta ahora no han sido consideradas. Estas acciones incluyen: la regulación de los usos del suelo presentes en una cuenca y su manejo (Lara et al. 2009), cambios en las prácticas de manejo del suelo (ejemplo: zanjas de infiltración que incrementan el almacenamiento de agua) y modificación de la composición, densidad y tamaño de los rodales boscosos, así como también mejoramiento en las prácticas de cosecha forestal y construcción de caminos en áreas donde se concentra la actividad forestal (Donoso 2009).

Un metaanálisis realizado para evaluar acciones de restauración ecológica sobre la recuperación de diversos servicios ecosistémicos ha demostrado un impacto positivo de tales acciones en servicios como la diversidad biológica, la fertilidad de suelos y la capacidad productiva de diferentes ecosistemas (Rey Benayas et al. 2009). Dentro de este contexto, las relaciones conocidas entre cobertura boscosa de las cuencas y la provisión de agua son la base para plantear la recuperación de este servicio ecosistémico. De esta manera, los principios y prácticas de la restauración definidos por SER (2009) pueden servir de guía conceptual para emprender proyectos en cuencas perturbadas, entendidas como ecosistemas a restaurar. Una imagen de referencia que integre aspectos ecológicoambientales, social-económicos y conocimiento-educación puede ser considerada como una efectiva forma de tener éxito (Palmer et al. 2005). En esta imagen de referencia, 
el hombre debe ser la pieza central, capaz de provocar cambios hacia una condición de recuperación, donde nuevamente interactúen elementos ecológicos y sociales, que otorguen una nueva y desconocida capacidad del ecosistema de adaptación o cambio (Walker et al. 2004, Shlüter y Pahl-Wostl 2007).

Los esfuerzos de restauración para la recuperación del servicio ecosistémico provisión de agua se justificarán en aquellas cuencas que han sufrido cambios como consecuencia del hombre y donde su impacto se ve reflejado en el reducido nivel de satisfacción de las distintas demandas sociales y económicas por dicho servicio. Ejemplos de ello son cuencas que entregan una deficiente calidad de agua que impacta la salud de la población, menores caudales que impiden y limitan el desarrollo de actividades productivas que dependen del uso del agua (agricultura, acuicultura, etc.), una mayor recurrencia e intensidad de eventos extremos (caudales mínimos, crecidas e inundaciones) que impactan la salud y bienestar de las personas, así como a la infraestructura.

El éxito de proyectos de restauración, enfocados en la recuperación del servicio ecosistémico provisión de agua, dependerá de un cambio sustancial de un determinado indicador incluido en la imagen de referencia. Así, por ejemplo, en los aspectos ecológicos y ambientales serán deseables cambios graduales y comprobables en la estructura de los ecosistemas a diferentes escalas espaciales para una mejor respuesta hidrológica de la cuenca (aumento de caudales bases, menor recurrencia e intensidad de eventos extremos). En el componente de los aspectos social y económico, un incremento en la satisfacción social y económica por el uso del agua genera una disminución de los conflictos asociados a este recurso. En el componente de conocimiento y educación, un avance sustancial en el conocimiento científico, difusión y transferencia tecnológica respecto a la ecología de cuencas y reeducación de su uso, y el desarrollo de políticas públicas expresadas, por ejemplo, en planes de ordenamiento territorial a escala de cuenca, monitoreo y aprendizaje para mejorar las prácticas de restauración.

La recuperación del servicio ecosistémico provisión de agua será uno de los temas más relevantes por resolver y un desafío para la investigación científica de los próximos años, especialmente dadas las predicciones de cambio climático para Chile, que indican una importante reducción de las precipitaciones (Trenberth et al. 2007). Conocer las tasas y plazos de recuperación, los límites de la oferta de este servicio ecosistémico, las acciones para mantenerlo, los costos involucrados e impacto social de esta recuperación, etc., será posible únicamente mediante experimentos de manipulación y seguimiento en el largo plazo. Un proyecto cuyo objetivo es conocer y entender cómo acciones de restauración ecológica impactan al servicio ecosistémico provisión de agua, está siendo implementado en la Reserva Costera Valdiviana. A partir del año 2006 se monitorea la calidad y cantidad de agua en 12 cuencas de pequeño y mediano tamaño. Algunas de ellas dominadas por plantaciones de Eucalyptus globulus Labill. serán reconvertidas a bosques nativos a través de ensayos de restauración mediante regeneración natural y artificial para evaluar la respuesta en la provisión de agua. Como referencia, Lara et al. (2009) predicen que por cada $10 \%$ que se logre recuperar la cobertura de bosques nativos será posible incrementar en un $14,1 \%$ los caudales totales de verano. Además, técnicas silviculturales que aceleran la sucesión ecológica, como por ejemplo raleos, podrían acortar los plazos de recuperación.

En estas mismas cuencas se desarrollan otros estudios y programas de educación sobre servicios ecosistémicos utilizando metodologías y enfoques transdisciplinarios (Lara et al. 2010), necesarios para buscar el éxito de la restauración ecológica, incluyendo aspectos económicos y sociales señalados en la imagen de referencia descrita anteriormente. Los resultados que se generen será de relevancia para el desarrollo de políticas públicas. Sin embargo, al igual que otros estudios ecológicos, donde existe la necesidad de evaluar simultáneamente una multidimensionalidad de elementos en el tiempo y espacio (Likens 1997, 2003), resolver las preguntas será un desafío para el trabajo transdisciplinario. Ello ofrecerá la oportunidad de conocer la resiliencia del ecosistema en un contexto social y los efectos de las acciones de restauración ecológica para la generación de nuevos conocimientos, enfoques y metodologías.

\section{AGRADECIMIENTOS}

Los autores agradecen el aporte del Núcleo Científico Milenio (P04-065-F), Fundación Centro de los Bosques Nativos, FORECOS; Fondecyt 1085024 y 1090479 ; CRN II 2047 del IAI, apoyado por la US NSF Grant GEO-0452325, y a CONICYT. A The Nature Conservancy, al equipo de gestión de la Reserva Costera Valdiviana (RCV) y la empresa forestal MASISA S.A. por el apoyo a la implementación y desarrollo de las cuencas experimentales en la RCV, como un sitio para estudios ecosistémicos de largo plazo.

\section{REFERENCIAS}

Angelstam P, J Roberge, T Ek, L Laestardius. 2005. Data and tools conservation, management, and restoration of northern forest ecosystems at multiple scales. In Stanturf J, P Madsen eds. Restoration of boreal and temperate forests. Florida, USA. CRC Press. p. 269-280.

Calder I. 1992. Hydrologic effects of land-use change. In Maindment DR ed. Handbook of Hydrology. New York, USA. McGraw-Hill. p. 13.1-13.5.

Donoso PJ. 2009. Tala rasa: implicancias y desafíos. Valdivia, Chile. Universidad Austral de Chile, Facultad de Ciencias Forestales. 98 p.

Echeverría C, D Coomes, J Salas, J Rey Benayas, A Lara, A Newton. 2006. Rapid deforestation and fragmentation 
of Chilean temperate forests. Biological Conservation 130: 481-494.

Farley K, E Jobbágy, R Jackson. 2005. Effect of afforestation on water yield: a global synthesis with implications for policy. Global Change Biology 11: 1565-1576.

Huber A, A Iroumé, J Bathurst. 2008. Effect of Pinus radiata plantation on water balance in Chile. Hydrological Process 22: $142-148$

Jackson, R. B., E Jobbagy, R Avissar, S Roy, D Barrett, C Cook, K Farley, D Le Maitre, B NcCarl, B Murray. 2005. Trading water for carbon with biological carbon sequestration. Science 210: 1944-1947.

Lara A, C Little, R Urrutia, J McPhee, C Álvarez-Garretón, C Oyarzún, D Soto, P Donoso, L Nahuelhual, M Pino, I Arismendi. 2009. Assessment of ecosystem services as an opportunity for the conservation and management of native forests in Chile. Forest Ecology and Management 258: 415-424.

Lara A, R Reyes, R Urrutia. 2010. Bosques nativos. Informe país. Estado del medio ambiente en Chile 2008. Santiago, Chile. Instituto de Asuntos Públicos. Centro de Análisis de Políticas Públicas, Universidad de Chile. p. 126-171.

Lara A, D Soto, J Armesto, P Donoso, C Wernli, L Nahuelhual, F Squeo eds. 2003. Componentes científicos clave para una política nacional sobre usos, servicios y conservación de los bosques nativos chilenos. Valdivia, Chile. Universidad Austral de Chile. 134 p. (Iniciativa Científica Milenio de Mideplan)

Lara A, R Villalba, R Urrutia. 2008. A 400-year tree-ring record of the Puelo river summer-fall streamflow in the Valdivian rainforest eco-region, Chile. Climatic Change 86: 331-356.

Likens GE. 1997. Limitation to intellectual progress in ecosystem science. In Pace M, M Groffman eds. Successes, Limitations and Frontiers in Ecosystem Science. New York, USA. Springer-Verlag. p. 247-271.

Likens G. 2003. Utilización de datos de largo plazo, balances de masa e isótopos estables en la bioquímica de cuencas. El modelo de Hubbard Brook. Gayana Botanica 60(1):3-7.

Little C, D Soto, A Lara, J Cuevas. 2008. Nitrogen exports at multiple-scales in a southern Chilean watershed (Patagonian Lakes district). Biogeochemistry 87(3): 297-309.

Little C, A Lara, J McPhee, R Urrutia. 2009. Revealing the impact of forest exotic plantations on water yield in large scale watersheds in South-Central Chile. Journal of Hydrology 374: 162-170.

Locatelli B, R Vignola. 2009. Managing watershed services of tropical forests and plantations: Can meta-analyses help? Forest Ecology and Management 258(9): 1864-1870.

Oyarzún C, A Huber. 1997. Nitrogen export from forested and agricultural watersheds of southern Chile. Gayana Botanica 60(1): 63-68.
Oyarzún C, L Nahuelhual, D Núñez. 2005. Los servicios ecosistémicos del bosque templado lluvioso: producción de agua y su valoración económica. Ambiente y Desarrollo 20: 88-95.

Oyarzún C, C Aracena, P Rutherford, R Godoy, A Deschrijver. 2007. Effects of land use conversion from native forests to exotic plantations on nitrogen and phosphorus retention in catchments of southern Chile. Water Air and Soil Pollution 179(1-4): 341-350.

Palmer M, E Bernhardt, J Allan, P Lake, G Alexander, S Brooks, J Carr, S Clayton, C Dahm, J Follstad, D Galat, S Loss, P Goodwin, D Hart, B Hassett, R Jenkinson, G Kondolf, R Lave, J Meyer, T O’Donnell, L Pagano, E Sudduth. 2005. Standards for ecologically successful river restoration. Journal of Applied Ecology 42: 208-217.

Rey Benayas JM, A Newton, A Diaz, J Bullock. 2009. Enhancement of biodiversity and ecosystem services by ecological restoration: A Meta-Analysis. Science 325: 1121-1124.

Schlüter M, C Pahl-Wostl. 2007. Mechanisms of resilience in common-pool resource management systems: an agent-based model of water use in a river basin. Ecology and Society 12(2):4 [online] URL: http://www.ecologyandsociety.org/ vol12/iss $2 /$ art $4 /$

Scott D, F Prinsloo. 2008. Longer-term effects of pine and eucalypt plantations on streamflow. Water Resources Research 44, W00A08, doi:10.1029/2007WR006781.

SER (Society for Ecological Restoration International-Sociedad Internacional para la Restauración Ecológica). 2004. Grupo de trabajo sobre ciencia y políticas. Principios de SER International sobre la restauración ecológica. Consultado 10 oct. 2009. Disponible en http://www.ser.org/content/ spanishprimer.asp

Trenberth KE, PD Jones, P Ambenje, R Bojariu, D Easterling, A Klein Tank, D Parker, F Rahimzadeh, JA Renwick, M Rusticucci, B Soden, P Zhai. 2007. Observations: Surface and atmospheric climate change. In Solomon S, D Qin, M Manning, Z Chen, M Marquis, KB Averyt, M Tignor and HL Miller eds. Climate Change 2007: The physical science basis. Contribution of Working Group I to the Fourth Assessment Report of the Intergovernmental Panel on Climate Change. Cambridge, United Kingdom and New York, NY, USA. Cambridge University Press.

Walker B, C Holling, S Carpenter, A Kinzig. 2004. Resilience, adaptability and transformability in social-ecological systems. Ecology and Society 9(2):5. [online] URL: http:// www.ecologyandsociety.org/vol9/iss 2/art5/

Ward A, S Trimble. 2003. Environmental hydrology. $2^{\text {nd }}$ ed. Florida, USA. CRC Press. 475 p.

Recibido: 14.01 .10

Aceptado: 09.06.10 